\title{
Unfavorable Effects of Fixatives on the Fluorescence Intensity and Biological Functions of Fluorescent Proteins in HEK293T Cells and Transgenic Mice
}

\author{
Tao Tang \\ Peking University People's Hospital \\ Lan Yuan \\ Peking University Health Science Center \\ Kai Wang ( $\nabla$ wang_kai@263.net) \\ Peking University People's Hospital \\ Mingwei Zhao \\ Peking University People's Hospital
}

\section{Research Article}

Keywords: fixatives, fluorescent proteins (FPs), fluorescence resonance energy transfer (FRET), transgenic fluorescent mice

Posted Date: August 18th, 2021

DOl: https://doi.org/10.21203/rs.3.rs-764380/v1

License: (c) (i) This work is licensed under a Creative Commons Attribution 4.0 International License. Read Full License 


\section{Abstract}

Fluorescent proteins (FPs) are commonly used probes for coding genes that enable specific protein or whole-cell labeling. Their fluorescence intensity is used for molecular quantitation and intermolecular interaction analysis. Since FPs are usually small soluble proteins, they easily cross the membranes if cell integrity is disrupted, resulting in FP signal attenuation/loss. Specimen prefixation to preserve FP localization within cells/tissues is therefore useful. However, specific fixatives can weaken or eliminate FP signals. We studied the effects of five common fixatives on FP fluorescence intensity and biological functions to determine their suitability for FP signal and FRET efficiency preservation in cells and tissues. FP (GFP, YFP, CFP and RFP)expressing HEK293T cells with methanol, 95\% ethanol, 4\% PFA, acetone and glutaraldehyde, and brain tissue sections of EGFPand tdTomato-labeled transgenic fluorescent mice was fixed with 4\% PFA. The FP signals in HEK293T cells and brain tissue from transgenic fluorescent mice were weakened or even eliminated after fixation with these fixatives. The fixatives affected FP biological function, and the FP FRET efficiency significantly differed between prefixation and postfixation (all $p<0.01$ ). Thus, fixatives impair FP fluorescence to some extent, leading to attenuation/loss of signals or even biological functions. Fixatives should be applied carefully in FP-related experiments to avoid bias.

\section{Introduction}

Fluorescent proteins (FPs) are commonly used reporter proteins in biology. They are often expressed in cells in the form of fusion proteins ${ }^{1-2}$. FPs, which are frequently used as probes for coding genes and can highlight proteins and cells of interest, are used in a wide range of applications, such as fluorescence imaging, fluorescence resonance energy transfer (FRET) techniques, and flow cytometry ${ }^{3-6}$. In addition, fluorescence in transgenic animals with the ability to spontaneously fluoresce upon excitation with light of the appropriate wavelength, such as transgenic fluorescent mice, provides scientists with a relatively simple and nontoxic biological tool and a tracing tool for cell therapy ${ }^{7-8}$. Before fluorescence imaging or FRET, it is generally necessary to fix cells or tissues with specific fixatives in order to maintain antigenicity and to prevent the FPs or cells from shifting during the experiment and affecting the downstream experimental results ${ }^{9}$. However, improper selection of fixatives for cells or tissues labeled with reporter FPs may lead to changes in the spatial conformations of FPs ${ }^{10}$. Such changes are problematic for FP detection, accurate localization and related microstructural analyses. Therefore, it is of practical significance to explore the influences of fixatives on FP signals.

FRET with FPs is a powerful method for the detection of protein-protein interactions, enzyme activity and small molecules in the intracellular milieu ${ }^{11-12}$, and it can also be used to determine the distance between two different fluorescent groups in real time $\mathrm{e}^{13-16}$. Accurate measurement of the increased fluorescence intensity of the acceptor group and the reduced fluorescence intensity of the donor group enables accurate quantification of the efficiency of FRET ${ }^{17}$. Living cells usually need to be fixed before FRET to preserve the cell structure and to prevent translocation of FPs and cells. In addition, for studies on transgenic fluorescent animals labeled with fluorescent reporter genes, which can be used to directly monitor cell activity and gene behavior in organisms with sensitive detection instruments, tissue fixation can help optimize sample preservation and the FP signal.

However, improper selection and use of fixatives may influence FP signals, affecting FP biological functions. In the current study, we applied five routinely used fixatives to investigate the effects of fixatives on the fluorescence intensity and FRET efficiency of FPs in HEK293T cells and transgenic fluorescent mice labeled with FPs and to further evaluate fixatives that produce minimal loss of FP fluorescence signals and biological functions in cells or tissues.

\section{Results}

HEK293T cells were infected with plasmids expressing GFP and RFP and with a lentivirus expressing YFP and CFP. Approximately $70-80 \%$ of cells revealed bright fluorescence under a confocal laser scanning microscope after culture for $48 \mathrm{~h}$. There was no obvious damage in the cells. Then, the cells expressing FPs were fixed at room temperature, and the changes in the FP fluorescence signals were detected under a confocal laser scanning microscope in real time (Fig. 1b, Fig. 2). 
The tested fixatives are used in research and have different stabilization mechanisms; thus, they have different effects on FP signals. Cells were postfixed with methanol, $95 \%$ ethanol, $4 \%$ paraformaldehyde (PFA), acetone and glutaraldehyde at room temperature. Over $10 \mathrm{~min}$, the YFP signal weakened rapidly, and with time, the YFP signal weakened further. Among cells fixed with methanol and 95\% ethanol, the YFP signal weakened significantly. Surprisingly, almost no YFP fluorescence signal was detectable after fixation with $4 \%$ PFA, while relatively strong fluorescence signals were preserved after fixation with acetone and glutaraldehyde. Postfixation with methanol and 4\% PFA significantly decreased CFP fluorescence, while a strong signal remained after postfixation with 95\% ethanol, acetone and glutaraldehyde. Both CFP fluorescence and YFP fluorescence were weakened after fixation with the five kinds of fixatives. The percentages of CFP and YFP fluorescence preservation were $14.71 \%$ and $19.27 \%$, respectively, after methanol fixation. The CFP and YFP fluorescence intensities after fixation with acetone were better than those after fixation with methanol (44.24\% and $62.64 \%$ ), respectively, and approximately $1 / 3$ of the CFP and YFP fluorescence intensity remained after glutaraldehyde fixation (Fig. 1, Table 1).

In the first 5 min of methanol, $95 \%$ ethanol and acetone fixation, the GFP and RFP fluorescence was reduced to less than $20 \%$ of the initial fluorescence, while $4 \%$ PFA fixation preserved more than $75 \%$ of the fluorescence. However, as the fixation time increased, the fluorescence signals of GFP and RFP decreased gradually. When the fixation time reached 15 min, the fluorescence intensities of GFP and RFP were $54.56 \%$ and $56.29 \%$ for the $4 \%$ PFA fixation group, respectively; the intensities were greater than $35 \%$ for the glutaraldehyde fixation group (Fig. 2, Table 1).

Table 1

Fluorescence intensity and fluorescence preservation percentages of FPs before and after fixation with five kinds of fixatives.

\begin{tabular}{|c|c|c|c|c|c|c|c|c|c|c|c|c|}
\hline \multirow[t]{2}{*}{$\begin{array}{l}\text { Fluorescent } \\
\text { proteins }\end{array}$} & \multicolumn{4}{|c|}{ Pre-fixation } & \multicolumn{4}{|c|}{ Post-fixation } & \multicolumn{4}{|c|}{$\begin{array}{l}\text { Fluorescence preservation } \\
\text { percentage }(\%)\end{array}$} \\
\hline & CFP & YFP & GFP & RFP & CFP & YFP & GFP & RFP & CFP & YFP & GFP & RFP \\
\hline Methanol & 26.14 & 27.23 & 25.41 & 25.85 & 3.85 & 5.25 & 3.43 & 3.67 & 14.71 & 19.27 & 13.50 & 14.20 \\
\hline $95 \%$ Ethanol & 27.81 & 20.44 & 25.46 & 25.42 & 11.65 & 5.98 & 3.76 & 1.29 & 41.89 & 29.26 & 14.41 & 5.07 \\
\hline 4\% PFA & 32.16 & 26.25 & 25.31 & 25.92 & 10.84 & 30.4 & 13.81 & 14.59 & 33.70 & 12.95 & 54.56 & 56.29 \\
\hline Acetone & 21.61 & 57.53 & 24.87 & 26.05 & 9.56 & 36.04 & 3.09 & 1.19 & 44.24 & 62.64 & 12.42 & 4.57 \\
\hline Glutaraldehyde & 26.05 & 26.05 & 25.08 & 25.54 & 8.03 & 8.02 & 9.20 & 10.19 & 30.83 & 30.79 & 36.68 & 39.90 \\
\hline
\end{tabular}

To test whether fixatives can also affect the biological functions of FPs by exerting unfavorable effects on FP signals, we performed FRET. As CFP and YFP are commonly used together as a pair, CFP and YFP were selected for the FRET experiments. Twenty-five independent CFP- and YFP-co-transfected cells were randomly selected for FRET from among living and fixed cells. There were significant differences in FRET efficiency between prefixation and postfixation with alcohol $(P=0.005), 95 \%$ ethanol $(P=0.001)$, acetone $(P=0.001), 4 \%$ PFA $(P<0.01)$ and glutaraldehyde $(P<0.01)$. The FRET efficiency increased when $95 \%$ ethanol was used; in contrast, it decreased when methanol, acetone, 4\% PFA and glutaraldehyde were used. Among the fixatives, the FRET efficiency of CFP and YFP decreased most obviously after fixation with 4\% PFA (Fig. 3).

Among all of the fixatives, 4\% PFA best preserved the fluorescence signals of GFP and RFP; thus, 4\% PFA was thus used to fix brain tissue sections of transgenic fluorescent mice labelled with EGFP and tdTomato. Figure 4 shows that the postfixation fluorescence intensities of brain sections were weaker than the prefixation intensities; however, there was no significant difference between pre- and postfixation $(p>0.05)$.

Taken together, these results show that fixatives unfavorably affect FP fluorescence in HEK293T cells and tissues of transgenic fluorescent mice to a certain extent and can thus lead to biased results regarding biological functions in analyses such as FRET.

\section{Discussion}


Methanol, 95\% ethanol, 4\% PFA, acetone and glutaraldehyde are routinely used as fixatives in biomedical experiments. In many studies, cells or tissues need to be fixed for subsequent microscopic observation, probe hybridization, or immunochemical staining. Before the relevant experiments are performed, the corresponding fixatives should be selected according to the specific cells, tissues and experimental purposes. FPs are applied as reporter genes and are widely used in gene transfection and expression studies and for in vivo fluorescence imaging of transgenic animals to observe the movement and localization of target proteins in cells. In addition, transgenic fluorescent animal models provide powerful tools for molecular imaging studies and studies on tumor biology, immune-tumor cell interaction, tumor angiogenesis, and stem cell differentiation and localization. Although FPs are widely applied in biomedical research, few studies have reported changes in the fluorescence intensity of FPs during cell or tissue fixation. Only sporadic information in relevant reports is available to researchers as guidance. Unfortunately, the descriptions of cell or tissue fixation methods in most studies are very simple, making it difficult for other experimenters to accurately determine the methods that were used. Therefore, the effects of some routinely used fixatives on the fluorescence intensity of FPs need to be elucidated.

Fixatives commonly applied in general molecular biology experiments are divided into two categories: organic solvents and crosslinkers $^{18}$. These fixatives are used for research and diagnostics and act through different stabilization mechanisms. The main organic solvent fixatives are methanol, ethanol and acetone. Methanol is a strong dehydrating agent that can immobilize cells in a certain configuration and increase the surface area of cell structures ${ }^{19}$. Ethanol is a small-molecule nonpolar permeabilizing substance that can penetrate cells quickly, fix the fine structures of cells, dehydrate tissues/cells, and denature proteins via precipitation to fix cells ${ }^{20}$. Acetone is a fixative with permeabilization, strong dehydration, and protein precipitation abilities $^{19}$. Paraformaldehyde and glutaraldehyde are routinely used crosslinking agents. The aldehyde group in the paraformaldehyde molecule binds with the amino groups of proteins to form carboxymethyl groups, thus forming intermolecular crosslinks that affect protein configurations and fix the proteins ${ }^{21}$. Glutaraldehyde is a bifunctional crosslinking agent $^{22}$ that has two reactive aldehyde groups that can undergo acylation with amino groups, mercapto groups and other groups, enabling it to crosslink with proteins. The changes in the molecular structure and conformation of cells induced by fixation may change the fluorescence intensity and protein interactions of FPs. Therefore, selection of an appropriate fixative is crucial for accurate detection of the protein of interest and its biological function.

Previous studies have reported that the GFP signal in GFP-expressing cells can be successfully maintained using PFA ${ }^{23-24}$. Our study showed that FP fluorescence decreased after fixation with 4\% PFA at room temperature and that CFP and exhibited the most significant fluorescence loss, while strong signals were preserved for GFP and RFP. According to some studies, FP signals decrease or disappear after fixation with $4 \%$ PFA; these findings may be related to deviation from a neutral $\mathrm{pH}$ during the preparation of $4 \%$ PFA. The $\mathrm{pH}$ value of $4 \%$ PFA in this experiment was close to neutral, so interference of the $\mathrm{pH}$ value with the experimental results was excluded. In another study with $4^{\circ} \mathrm{C}$ PFA, the GFP signal was barely detectable, indicating that crosslinking of proteins was too slow to retain GFP; in contrast, more confined and intense GFP signals were obtained in sections postfixed with $25^{\circ} \mathrm{C}$ PFA ${ }^{25}$. Interestingly, another fixative called glyoxal has recently been proposed to be a relevant alternative to PFA, but whether glyoxal works as a postfixative to effectively preserve FP signals remains to be investigated. Few studies have reported the effect of glutaraldehyde on FP signals. In this study, glutaraldehyde also decreased FP fluorescence; approximately $1 / 3$ of the fluorescence was preserved.

Previous studies have shown that acetone can preserve GFP signals as well. The results of our study showed that acetone weakened the FP signals, among which the GFP and RFP signals decreased most obviously. However, the CFP and YFP signals remained strong. The different mechanisms of acetone and paraformaldehyde fixation in cells provide choices for experimental studies using different FPs as gene probes.

Some studies have reported that methanol can also be applied to maintain GFP fluorescence ${ }^{26}$, but our study showed that methanol may lead to weakening or even disappearance of GFP fluorescence ${ }^{10}$. In fact, the fluorescence signals of all four FPs decreased significantly after fixation with methanol, indicating that methanol could not effectively preserve the FP signals. Therefore, methanol is not recommended for fixation of cells expressing FPs. 
Ethanol-mediated cell fixation has been found to cause GFP fluorescence to disappear quickly ${ }^{5}$. In agreement with this finding, our study revealed that the fluorescence intensity of the four FPs was weakened rapidly after fixation with $95 \%$ ethanol. Among the signals, the RFP signal was lost most obviously, while some of the fluorescence intensity of CFP was preserved. Some studies have reported that methanol and ethanol alone or mixed with acetone and formaldehyde can be used as fixatives. However, unless otherwise required for specific experiments, for the types of FPs applied in our study, acetone is recommended for fixation to maintain CFP and YFP fluorescence, while 4\% PFA is recommended for fixation to preserve GFP and YFP fluorescence.

Another question is whether increases or decreases in FP fluorescence during fixation will affect the results of related experiments based on the fluorescence intensity of FPs. To answer this question, we selected two interacting proteins, ADORA2a and DRD2, for co-expression with YFP and CFP, respectively. We co-transfected them into HEK293T cells with lentiviruses and then detected the FRET efficiency. The optimum distance of FRET detection is $1-10 \mathrm{~nm}$; when the spatial displacement of FP molecules is too large, it affects the detection efficiency of FRET, especially for cells with strong molecular motion of FPs. In such cases, it is necessary to fix the cells in order to retain the interactions between protein molecules in a particular cell state and to perform the FRET experiment smoothly.

In our study, the fixatives had significant negative impacts on FRET efficiency (all P< 0.01 ). The FRET efficiency after $95 \%$ ethanol fixation was higher than that before fixation, while the efficiencies after methanol, 4\% PFA, acetone and glutaraldehyde fixation were all lower than those before fixation. The FRET efficiency decreased most obviously after fixation with $4 \%$ PFA. Previous studies have shown no significant differences in FRET efficiency between unfixed living cells and living cells fixed with $4 \%$ PFA $^{27}$. Chu Y W et al. ${ }^{24}$ showed that FRET efficiency decreased after PFA fixation, consistent with the results of this study. The effects may be related to the mechanisms of the fixatives; the specific mechanisms need further study.

This experiment also used 4\% PFA-fixed brain tissue sections of transgenic mice labeled with EGFP and Ai4 transgenic mice labeled with tdTomato to explore the effect of fixatives on FP signals. The results showed that the fluorescence signals of EGFP and tdTomato in transgenic fluorescent mouse brain tissues decreased after fixation with $4 \%$ PFA.

According to the results of our study, the effects of fixatives on FP signals in cultured cells and transgenic fluorescent mouse tissues are unfavorable to some extent. Our findings also confirmed that these effects can cause bias in FRET efficiency. Whether the effects also cause errors in the results of other experiments based on the fluorescence intensities of FPs, such as flow cytometry or immunohistochemistry, remains to be examined.

Taken together, our findings reveal that fixatives indeed have certain unfavorable effects on FP signals in cells and tissues that lead to decreases in or even loss of their biological functions. Therefore, fixatives should be applied carefully in FP-related experiments to avoid bias in the experimental results.

\section{Methods}

\section{Cell culture and transfection.}

HEK293T cells, which was purchased from ATCC, were cultivated on 6 -well plate (Nunc) at $37^{\circ} \mathrm{C}$ in $95 \%$ air and $5 \% \mathrm{CO}_{2}$. The medium was DMEM (Gibco, Invitrogen Corporation, NY, United States) containing 10\% fetal bovine serum (FBS, Gibco), 100 units $/ \mathrm{ml}$ penicillin, and $100 \mu \mathrm{g} / \mathrm{ml}$ streptomycin (Gibco). The cells were transfected 1 day after cultivating, at which time they were $80 \%$ confluent. The cells on a 6-well plate were transfected with a mixture of $1 \mathrm{mg}$ of plasmid DNA encoding four kinds of FP-fusion constructs and $12 \mathrm{mg}$ of Lipofectamine reagent (Gibco BRL, Gaithersburg, MD) in a 0.2-ml vol of Opti-MEM (Gibco $\mathrm{BRL}$ ). After 5 hours, the transfection mixture was replaced with $1 \mathrm{ml}$ of culture medium. The cells were used 2 days after transient transfection. The fluorescence of the cells was visible, and $70-80 \%$ of the cells exhibited bright fluorescence under confocal laser scanning microscopy. These infected cells were then prepared for the experiments. Cells without plasmid or lentivirus infection were used as negative controls. All processes in this study involving plasmid or lentivirus were performed in a biosafety level 3 (BSL-3) facility. 


\section{Cell processing.}

The cell culture medium was removed, and the cells were washed with phosphate buffer saline (PBS) 3 times. The fluorescence of living cells was observed under a confocal laser scanning microscope. The method of acceptor photobleaching (acceptor bleaching, AB) was applied to detect the fluorescence resonance energy transfer (FRET) efficiency between CFP and YFP. The cells were first fixed at room temperature for $10 \mathrm{~min}^{5}$ using five kinds of fixatives and then diluted with $4 \mathrm{ml}$ sterile water. The changes in FP signals were detected by confocal laser scanning microscopy in real time. The monitoring time was 15 min in total. After $15 \mathrm{~min}$, the fixatives were discarded, and the cells were gently and carefully washed 4 times with PBS. The FRET experiment was then conducted again as post-fixative results.

\section{Ethics statements.}

EGFP- and tdTomato- expressing transgenic mice, which were purchased from the Jackson Laboratory, were used for the experiment. For studies on transgenic mice, we confirm that all methods were carried out following relevant guidelines and regulations. Furthermore, we confirmed that the study was carried out in compliance with the ARRIVE guidelines. All mice in the study were maintained and used according to protocols approved by the Institutional Animal Care and Use Committee of the Peking University Health Science Center (Ethics number: LA2020310). The mice housed in Transgenic Biosafety BSL-3 laboratories of the Peking University Health Science Center Laboratory Animal Application and Research Center. All processes of the animal experiment were in line with recommendations for the care and use of laboratory animals.

\section{Tissue processing.}

The EGFP and tdTomato expressing transgenic fluorescent mice were sacrificed according to normal pathological procedures. Briefly, mice were sacrificed by intraperitoneal injection of pentobarbital sodium (150mg/kg). Mouse brains were dissected and snap-frozen sliced at a 14 um thickness, and then placed brain sections under a confocal laser scanning microscope to observe the fluorescence intensities. After recording the data, the brain sections were fixed with $4 \%$ paraformaldehyde (PFA) for 12 hours. After 3 washes with PBS, the sections were observed under a confocal laser scanning microscope to record the fluorescence intensities again.

\section{Confocal microscopy.}

Cells and tissues were visualized using a confocal laser scanning microscope (Leitz Co.; with Technical Instruments coaxialconfocal attachment). The cells were viewed using a 100x oil immersion objective, and the tissues were viewed using a $40 \times$ objective.

\section{Statistics.}

Graphing and statistical analyses were performed using GraphPad Prism 8 software (version 8.0.2, GraphPad Software Inc.) and SPSS 24.0 software (version 24.0, IBM Corp., US).

Paired-sample t tests were used to analyze the two groups of data. $\mathrm{P}<0.05$ was considered to indicate a significant difference.

\section{Declarations}

\section{Foundation projects:}

National Natural Science Foundation of China ((Grant No. 81870684 \& 91630314), the Non-Profit Central Research Institute Fund of the Chinese Academy of Medicine Sciences (Grant No. 2019HY320001), The National Key Research and Development 


\section{Acknowledgements}

None.

\section{Author contributions}

Lan Yuan and Kai Wang developed the concept and designed the experiments. Tao Tang performed the experiments and the data analysis. Tao Tang wrote the manuscript. Mingwei Zhao developed the concept. All authors reviewed the manuscript.

\section{Competing interests}

The authors have no conflicts of interest to disclose and no proprietary interests in any of the materials mentioned in this article.

\section{References}

1. Farrants, H. et al. Chemogenetic Control of Nanobodies. Nat. Methods, 17, 279-282 (2020).

2. Stewart, M. P., Helenius, J., Toyoda, Y., Ramanathan, S. P. \& Muller, D. J. \& Hyman A.A. Hydrostatic pressure and the actomyosin cortex drive mitotic cell rounding. Nature, 469, 226-230 (2011).

3. Patriarchi, T. et al. An expanded palette of dopamine sensors for multiplex imaging in vivo. Nat Methods, 17, 1147-1155 (2020).

4. Strack, R. GFP lights up amyloid fibrils. Nat Methods, 17, 959 (2020).

5. Stapornwongkul, K. et al. Patterning and growth control in vivo by an engineered GFP gradient., 370, 321-327 (2020).

6. Motazedian, A. et al. Multipotent RAG1 + progenitors emerge directly from haemogenic endothelium in human pluripotent stem cell-derived haematopoietic organoids. Nat Cell Biol, 22, 60-73 (2020).

7. Kaiser, T., Ting, J. T., Monteiro, P. \& Feng, G. Transgenic labeling of parvalbumin-expressing neurons with tdTomato., 321, 236-245 (2016).

8. Chishti, A. A. et al. Constitutive expression of tdTomato protein as a cytotoxicity and proliferation maker for space radiation biology. Life Sci Space Res (Amst), 4, 35-45 (2015).

9. Poll, S. et al. Memory trace interference impairs recall in a mouse model of Alzheimer's disease. Nat Neurosci, 23, 952-958 (2020).

10. Carraway, K. L., Carraway, C. C. \& Cytoskeleton Signalling and Cell Regulation: A Practical Approach114(Oxford University Press, Oxford, 2000).

11. Wu, L. et al. Forster resonance energy transfer (FRET)-based small-molecule sensors and imaging agents. Chem Soc Rev, 49, 5110-5139 (2020).

12. Ai, H. W., Hazelwood, K. L., Davidson, M. W. \& Campbell, R. E. Fluorescent protein FRET pairs for ratiometric imaging of dual biosensors. Nat.Methods, 5, 401-403 (2008).

13. Fan, J., Hu, M., Zhan, P. \& Peng, X. Energy transfer cassettes based on organic fluorophores: construction and applications in ratiometric sensing. Chem Soc Rev, 42, 29-43 (2013).

14. Yuan, L., Lin, W., Zheng, K. \& Zhu, S. FRET-based small-molecule fluorescent probes: rational design and bioimaging applications. Acc Chem Res, 46, 1462-1473 (2013).

15. Algar, W. R., Hildebrandt, N., Vogel, S. S. \& Medintz I.L. FRET as a biomolecular research tool -understanding its potential while avoiding pitfalls. Nat Methods, 16, 815-829 (2019). 
16. Hoetelmans, R. W. et al. Effects of acetone, methanol, or paraformaldehyde on cellular structure, visualized by reflection contrast microscopy and transmission and scanning electron microscopy. Appl Immunohistochem Mol Morphol, 9, 346351 (2019).

17. Stryer, L. \& Haugland, R. P. Energy transfer: a spectroscopic ruler. Proc Natl Acad Sci U S A, 58, 719-726 (1967).

18. Takahara, T. et al. Diffusion weighted whole body imaging with background body signal suppression (DWIBS): technical improvement using free breathing, STIR and high resolution 3D display. Radiat Med, 22, 275-282 (2004).

19. Pollice, A. A. et al. Sequential paraformaldehyde and methanol fixation for simultaneous flow cytometric analysis of DNA, cell surface proteins, and intracellular proteins., 13, 432-444 (1992).

20. Shi, S. R. et al. Evaluation of the value of frozen tissue section used as "gold standard" for immunohistochemistry. Am J Clin Pathol, 129, 358-366 (2008).

21. DiDonato, D. \& Brasaemle, D. L. Fixation methods for the study of lipid droplets by immunofluorescence microscopy. $J$ Histochem Cytochem, 51, 773-780 (2003).

22. Szwarocka, A. \& Jozwiak, Z. The effect of daunorubicin and glutaraldehyde treatment on the structure of erythrocyte membrane. International Journal of Pharmaceutics, 181, 117-123 (1999).

23. Dreyfus, P. A. et al. Adult bone marrow-derived stem cells in muscle connective tissue and satellite cell niches. Am J Pathol, 164, 773-779 (2004).

24. Chu, Y. W., Wang, R., Schmid, I. \& Sakamoto, K. M. Analysis with flow cytometry of green fluorescent protein expression in leukemic cells., 36, 333-339 (1999).

25. Scandella, V., Paolicelli, R. C. \& Knobloch, M. A novel protocol to detect green fluorescent protein in unfixed, snap-frozen tissue. Sci Rep, 10, 14642 (2020).

26. Girotti, M. \& Banting, G. TGN38-green fluorescent protein hybrid proteins expressed in stably transfected eukaryotic cells provide a tool for the real-time, in vivo study of membrane traffic pathways and suggest a possible role for ratTGN38. J Cell Sci, 109, 2915-2926 (1996).

27. Marx, V. \& Probes FRET sensor design and optimization. Nat.Methods, 14, 949-953 (2017).

\section{Figures}



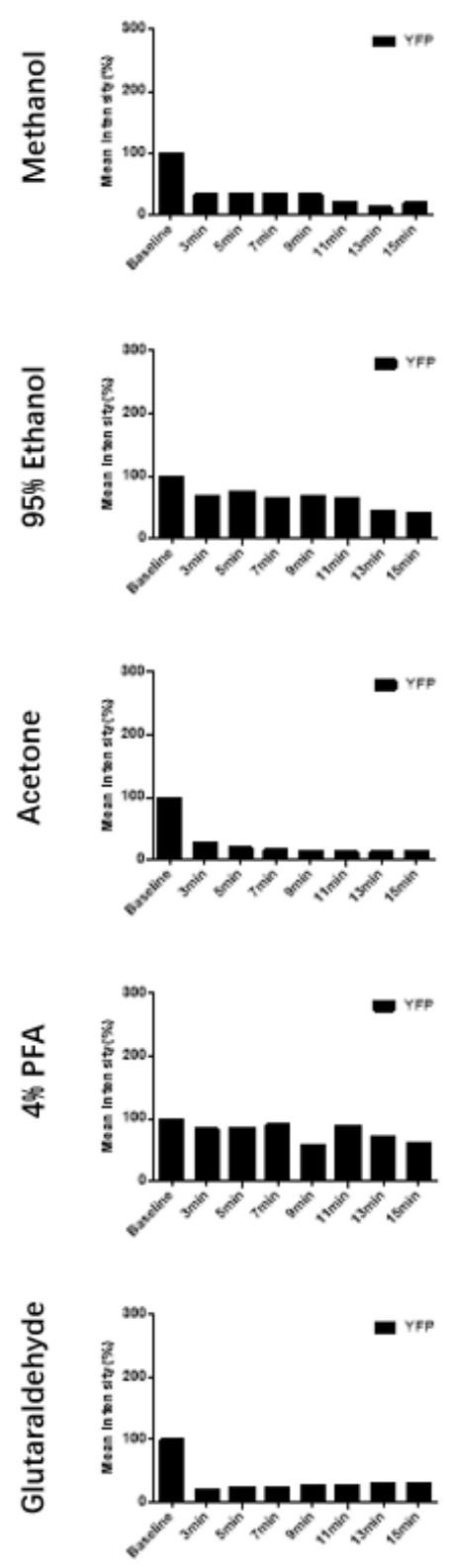
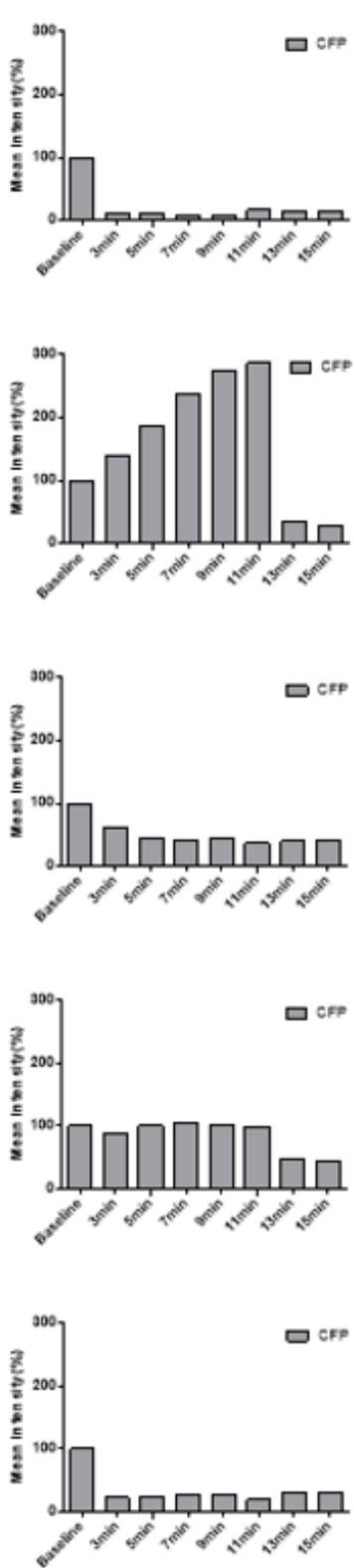

a
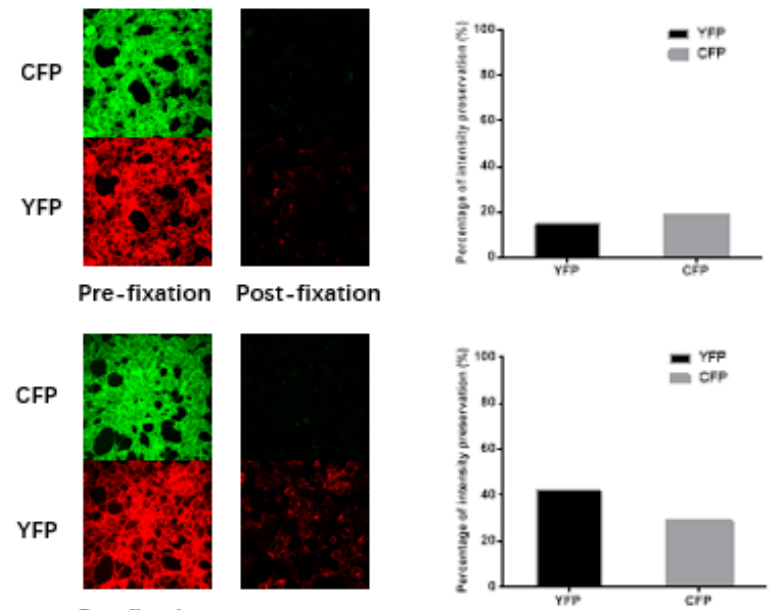

Pre-fixation Post-fixation
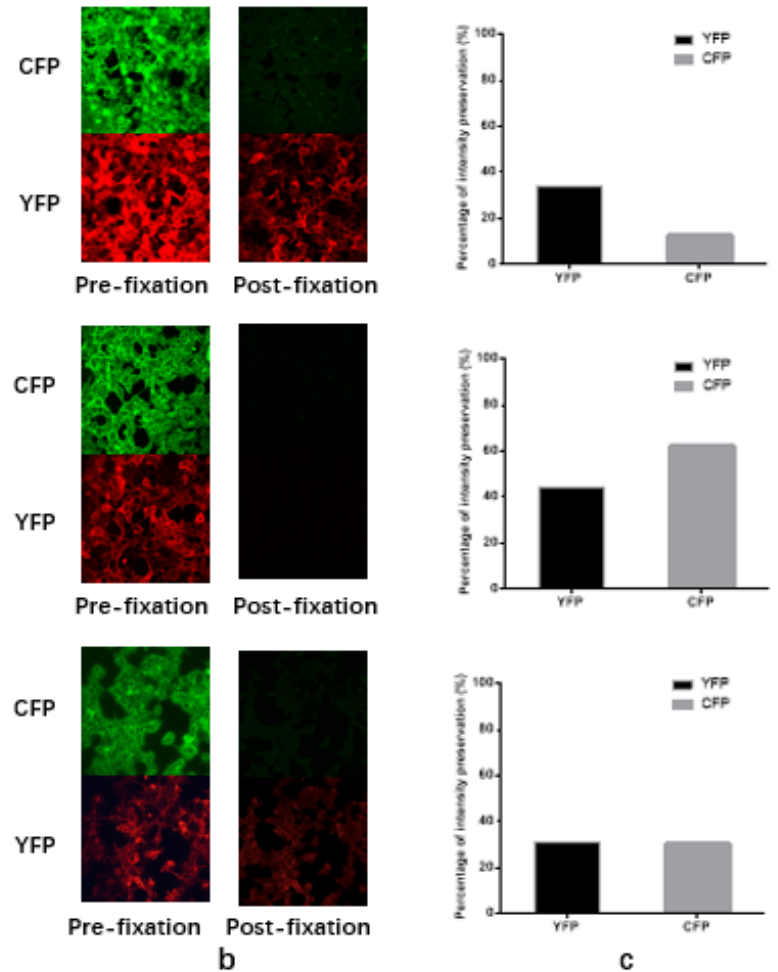

Figure 1

Effects of five kinds of fixatives on YFP and CFP fluorescence signals detected under a confocal laser scanning microscope in real time. (a) Quantification of YFP and CFP fluorescence signals after fixation with five kinds of fixatives. The bar graph shows the percentages of YFP and CFP fluorescence signals normalized to the prefixation signal. (b) YFP (red) and CFP (green) fluorescence observed under a confocal laser scanning microscope at prefixation and postfixation. Both YFP and CFP fluorescence signals were weakened after fixation with the five kinds of fixatives. (c) Percentage (\%) of YFP and CFP fluorescence preserved by methanol, $95 \%$ ethanol, 4\% PFA, acetone and glutaraldehyde. 

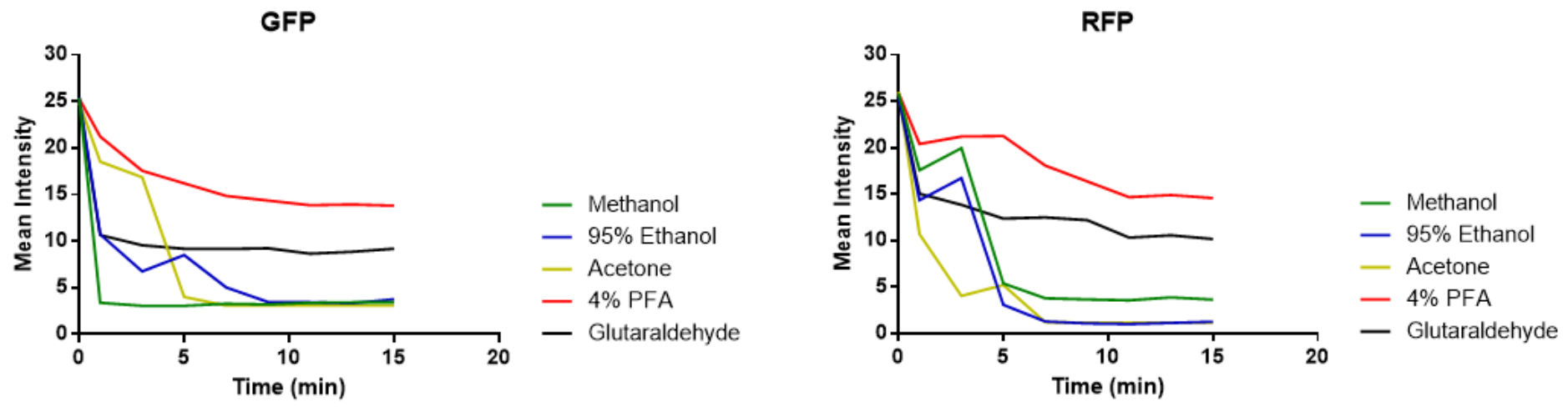

Figure 2

Dynamic curves of GFP (left) and RFP (right) fluorescence intensity in HEK293T cells fixed with methanol, 95\% ethanol, 4\% PFA, acetone and glutaraldehyde.

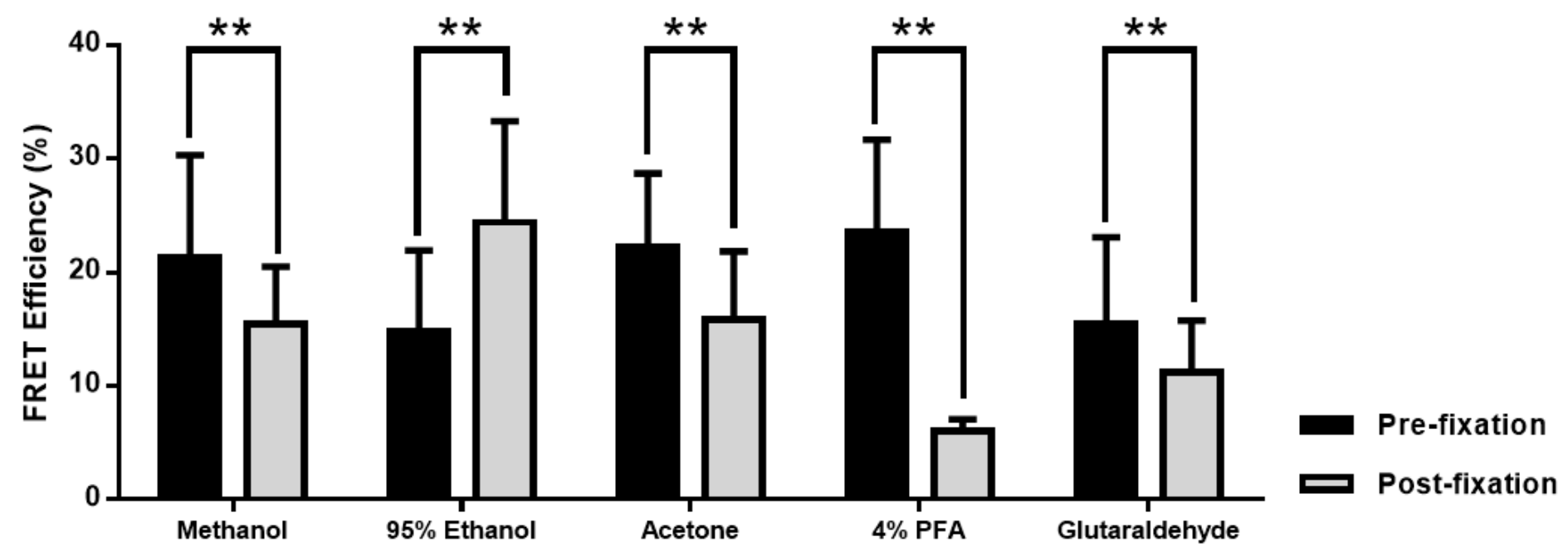

Figure 3

Effects of five kinds of fixatives on the FRET efficiency of CFP and YFP before and after fixation. $* * P<0.01$ was considered to indicate a significant difference. 


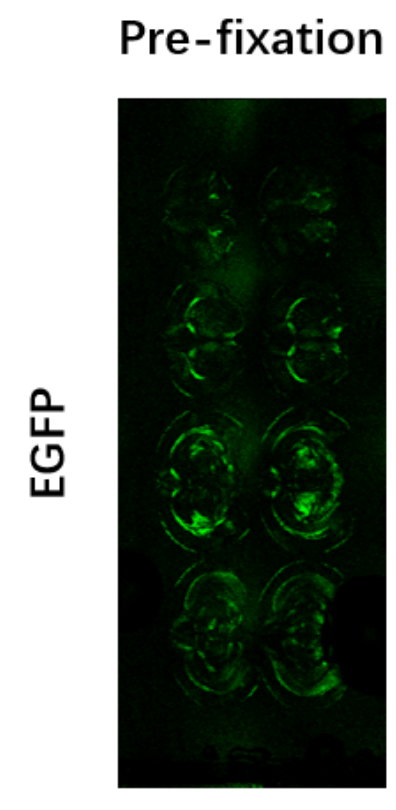

\section{Post-fixation}
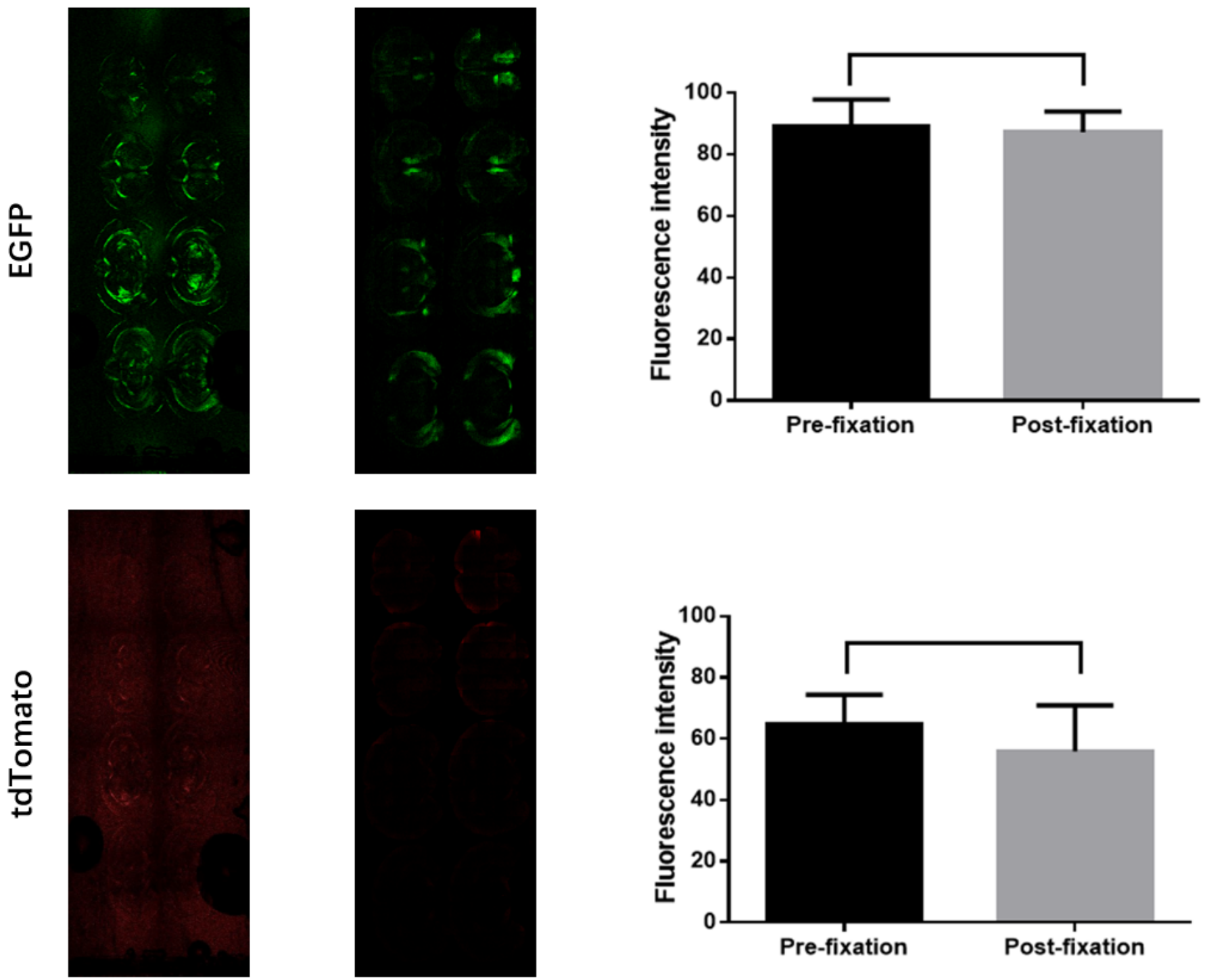

a

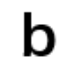

Figure 4

Decreased fluorescence intensity of brain tissue sections of transgenic fluorescent mice labelled with EGFP and tdTomato and fixed with 4\% PFA. (a) Fluorescence intensity of EGFP and tdTomato before and after fixation with 4\% PFA. (b) Bar graph of the fluorescence intensity (mean $\pm S D, n=5$ sections). 